\title{
The response of adipose tissues to Mycoplasma pulmonis and Sendai virus infection in C57BL/6 and DBA/2 mice
}

\author{
Tussapon BOONYARATTANASOONTHORN ${ }^{1,2}$, Keisuke SATO ${ }^{1)}$, \\ Yuko OKAMATSU-OGURA ${ }^{3)}$, Masami MORIMATSU ${ }^{1)}$ and Takashi AGUI ${ }^{1 \text { )* }}$ \\ 1)Laboratory of Laboratory Animal Science and Medicine, Department of Applied Veterinary Sciences, \\ Graduate School of Veterinary Medicine, Hokkaido University, Hokkaido 060-0818, Japan \\ ${ }^{2)}$ Department of Physiology, Faculty of Veterinary Science, Chulalongkorn University, Bangkok, 10330, Thailand \\ 3) Laboratory of Biochemistry, Department of Basic Veterinary Sciences, Graduate School of Veterinary Medicine, \\ Hokkaido University, Hokkaido 060-0818, Japan
}

\section{J. Vet. Med. Sci.}

83(3): 403-411, 2021

doi: $10.1292 /$ jvms.20-0625

Received: 27 November 2020 Accepted: 6 January 2021 Advanced Epub: 25 January 2021

\begin{abstract}
Adipose tissues in mammals are categorized into white and brown adipose tissues in which cellular morphology, cell functions, and tissue distribution are different. White adipose tissue (WAT) plays a major role in energy reservation, while brown adipose tissue (BAT) mainly relates to the thermoregulation of the body. One interesting function of adipose tissue is the response to the infection, especially the pathogens that cause pneumonia. We have previously reported that DBA/2 (D2) mice are susceptible to pathogens causing pneumonia, Mycoplasma (M.) pulmonis and Sendai virus (SeV), whereas C57BL/6 (B6) mice are resistant to them. Furthermore, morphological alteration of mediastinal fat tissue (MFT) was seen after infection of $M$. pulmonis in D2 mice but not in B6 mice. In this study, we aimed to exhibit the difference in adipose tissue response in other areas, including interscapular brown adipose tissue (iBAT), inguinal white adipose tissue (ingWAT), and perigonadal WAT (perigoWAT) between resistant strain, B6 and susceptible strain, D2 after challenging them with M. pulmonis and SeV. Compared with B6 mice, D2 mice showed an increase in fat-associated lymphoid cluster in MFT, an increase in BAT in both iBAT and ingWAT after M. pulmonis and SeV infection. The results of this study indicate that pneumonia caused by M. pulmonis and SeV infection induces browning of adipocyte, suggesting that BAT plays a role in pathogen infection and inflammation.
\end{abstract}

KEY WORDS: adipose tissue, brown adipose tissue, Mycoplasma pulmonis, Sendai virus, uncoupling protein 1

Adipose tissue is known as the largest endocrine organ consisting of adipocytes, fibroblasts, macrophages, and endothelium [23]. The main function of adipose tissue is not only a place for triglycerides storage but also contribution to a variety of physiologic functions, for example, energy balance and immune reactions $[1,4,7,10,18,22]$. Approximately $70 \%$ of the stromal vascular fraction of adipose tissue consists of immune cells including macrophages, mast cells, neutrophils, and lymphocytes. These cells participate in whole-body immune signaling via secretion of adipokines and cytokines such as adiponectin, tumor necrosis factor (TNF)- $\alpha$, interferon (IFN)- $\gamma$, and interleukin (IL)-6 [3]. Moreover, white adipose tissue (WAT), brown adipose tissue (BAT), and adipocytes are targets of many pathogens such as Trypanosoma cruzi [15], Staphylococcus aureus [25], Mycobacterium canettii [6], and Mycobacterium tuberculosis [3], resulting in a change of adipose tissue. However, the function of adipose tissue in the progression and pathology of pathogens during infection is remained unclear.

Several studies using different inbred mouse strains showed various susceptibilities to the pathological infection. For example, DBA/2 (D2) mice had greater proliferation of pathogens in the lung, higher infiltration of immune cells such as neutrophils, macrophages, and lymphocytes, and more production of inflammatory cytokines: IL- $1 \beta$, IL- 6 , IL-17A, and TNF- $\alpha$ in bronchoalveolar lavage (BALF) fluid than did C57BL/6 (B6) mice in the infection of pathogens that cause pneumonia such as Sendai virus (SeV) [27] and Mycoplasma (M.) pulmonis [5]. Results from our previous study showed differences between B6 and D2 in increasing fat-associated lymphoid clusters (FALCs) in mediastinal fat tissues (MFTs) after infected with M. pulmonis. Therefore, we continue to investigate the role of adipose tissue that might be associated with proliferation of pathogens, innate and adaptive immune response, inflammatory cell infiltration, and severe body weight reduction. This information would contribute to 
the discovery of the interaction between pathogens and adipose tissue progression in infectious diseases.

Thus, in the current study, we examined the response in adipose tissue to M. pulmonis and SeV infection in B6 (resistant) and D2 (susceptible) mice, to exhibit the profiling of the transformation of adipose tissue by observing the amount of BAT from three different adipose tissue depots. The result showed D2 mice steadily had a much greater amount of BAT in interscapular BAT (iBAT) and inguinal WAT (ingWAT) during the infection. This study is the first report to identify differential responses of BAT and WAT in response to M. pulmonis and $\mathrm{SeV}$ infection in mice.

\section{MATERIALS AND METHODS}

\section{Mice and tissue sampling}

Female C57BL/6NCrSlc (B6) and DBA/2CrSlc (D2) mice, 8-week-old, were obtained from Japan SLC (Hamamatsu, Japan). All mice were maintained under the specific pathogen-free conditions and infection experiments were conducted in the bio-safety level 3 facility. They were given access to sterile food and water ad libitum before and during experiments. Animal experimentation was conducted under the animal care and use program accredited by AAALAC International. Animal use protocol was approved by the President of Hokkaido University after review by the Institutional Animal Care and Use Committee (approval number 18-0169). After euthanizing the mice by inhalation of an overdose of isoflurane (Pfizer, Tokyo, Japan), MFT, iBAT, ingWAT, and perigonadal WAT (perigoWAT) were quickly removed and transferred into RNAlater storage solution (Thermo Fisher Scientific, Gaithersburg, MD, USA) for quantitative PCR analysis and 4\% paraformaldehyde for histological examination.

\section{Bacteria and infection}

The CIEA-NH strain of M. pulmonis was obtained from Dr. Nobuhito Hayashimoto (Central Institute for Experimental Animals, Kawasaki, Japan) and cultured in mycoplasma broth as previously described [5]. Briefly, mycoplasma broth base (BBL Microbiology Systems, Cockeysville, MD, USA) was mixed with D (+)-glucose (Wako Pure Chemical Industries, Ltd., Osaka, Japan) and phenol red (Wako), then dissolved in distilled water and autoclaved. After cooling the autoclaved broth, heat-inactivated horse serum (GIBCO Laboratories, Grand Island, NY, USA), 25\% fresh yeast extracts (Oriental Yeast Co., Ltd., Tokyo, Japan), $2.5 \%$ thallium acetate (Wako), and ampicillin sodium salt (Sigma Chemical Co., St. Louis, MO, USA) were added. The propagated M. pulmonis was divided into $1-\mathrm{ml}$ aliquots and frozen at $-80^{\circ} \mathrm{C}$ as stock cultures until used. On the day of infection, mice from each strain $(n=4-6)$ were anesthetized by inhalation of isoflurane, followed by intraperitoneal injection of the combination of anesthesia drugs as described previously [12], $30 \mu \mathrm{l}$ of inoculum containing $6.0 \times 10^{5} \mathrm{CFU}$ of M. pulmonis were intranasally inoculated. The same volume of mycoplasma broth alone was given to control group from each strain after the same condition of anesthetization. During the infection, clinical signs, body temperature, and body weight were observed daily. At 14 days-post infection (d.p.i.) all mice were euthanized by inhalation of an overdose of isoflurane to perform the sample collection.

\section{Virus and infection}

The MN strain of $\mathrm{SeV}$ was propagated and preserved in modified Eagle medium (Sigma) with 1\% bovine serum albumin as stock. The stock culture was inoculated into 10-day-old embryonated chicken eggs and incubated at $35^{\circ} \mathrm{C}$ for $72 \mathrm{hr}$. The allantoic fluid was centrifuged at $2,500 \times g$ for $20 \mathrm{~min}$, and then the supernatant was taken for infection experiment and stored at $-80^{\circ} \mathrm{C}$ until used. Determination of virus titer in the allantoic fluid was performed under hemadsorption assay by using a monkey kidney cell line, LLC-MK2, and chicken red blood cells, then median tissue culture infectious dose $\left(\mathrm{TCID}_{50}\right)$ was calculated for indication of the viral titer value. Mice from each strain $(n=4-6)$ were anesthetized by inhalation with isoflurane, followed by intraperitoneal injection of the combination of anesthesia drugs as described previously [12]. Then, $25 \mu 1$ of the inoculum of $10^{3} \mathrm{TCID}_{50} \mathrm{SeV}$ was inoculated intranasally to mice as previously described [26]. The same volume of modified Eagle medium alone was given to control group from each strain after the same condition of anesthetization. During the infection, the main symptoms for SeV infection, for example, respiratory distress, lethargy, and hunched posture were observed daily by visual observation as well as the body weight of mice was recorded. All mice were euthanized at 8 d.p.i. by inhalation of an overdose of isoflurane, and then sample collection was performed.

\section{Adipose tissue histopathology}

Adipose tissues were removed, fixed overnight in 4\% paraformaldehyde, and then washed in distilled water. After washing specimens were transferred to graded alcohol and embedded in paraffin. The paraffin-embedded specimens were cut into $3 \mu \mathrm{m}-$ thick sections, subsequently deparaffinized, rehydrated, and stained with haematoxylin and eosin (HE). The stained sections were examined under a light microscope.

To obtain the light micrographs of the stained sections, a NanoZoomer-XR Digital slide scanner (Hamamatsu Photonics K.K., Hamamatsu, Japan) was used. The light micrographs of each sample were used for measuring the area interested by using NDP. view2 Viewing software (ver. 2.6.13, Hamamatsu Photonics). For the MFT light micrographs, the area of FALC, the area of BAT, and the total area of the mediastinal white adipose tissue were measured, and the ratio of FALC area to total MFT area and the ratio of BAT area to total MFT area were calculated. For the iBAT, ingWAT, and perigoWAT light micrographs, the area of BAT and the total areas of iBAT, ingWAT, and perigoWAT were measured using the same software as above, and then the ratio of BAT area to total iBAT area, total ingWAT area, and total perigoWAT area were calculated. 


\section{$m R N A$ analysis}

The RNAiso reagent (Takara Bio, Kusatsu, Japan) was used for RNA extraction from the adipose tissue following to the manufacturer's protocol. The quality and quantity of the purified RNA were assessed by a NanoDrop instrument (NanoDrop Products, Wilmington, DE, USA). Total RNA $(2 \mu \mathrm{g})$ was reverse-transcribed using a 15 -mer oligo (dT) adaptor primer and M-MLV reverse transcriptase (Promega, Madison, WI, USA). Real-time PCR was performed on a fluorescence thermal cycler (LightCycler system, Roche Diagnostics, Mannheim, Germany) using FastStart Essential DNA Green Master (Roche Diagnostics) following to the manufacturer's protocol. The mRNA expression levels of Ucpl gene in adipose tissues were determined using the standard $U c p 1$ cDNA fragments to make a standard curve and Actb mRNA as internal standard for data normalization. The primers used in this study were as follows: 5'-TCGTACCACAGGCATTGTGAT-3' and 5'-TGCTCGAAGTCTAGAGCAAC-3' for Actb (NCBI accession number NC_000071.7), 5'-GGCCTCTACGACTCAGTCCA-3' and 5'-TAAGCCGGCTGAGATCTTGT-3' for Ucp 1 (NCBI accession number NC_000074.7).

\section{Measurement of body temperature}

The mouse body temperature was measured at pre- and post-infection using an infrared thermometer, Famidoc, Harasawa Pharmaceutical Co., Ltd. (Tokyo, Japan) by contacting the probe with abdominal skin.

\section{Statistical analysis}

All values are expressed as the mean \pm standard error of the mean (SE). Statistical analyses were performed by using an analysis of variance (ANOVA) followed by Scheffe's post-hoc test. Values of $P \leq 0.05$ were considered being statistically significant.

\section{RESULTS}

\section{Development of FALCs and BAT in MFTs in infected B6 and D2 mice}

B6 and D2 mice ( $\mathrm{n}=4-6$ per strain) were infected with $M$. pulmonis and $\mathrm{SeV}$ to examine the development of FALC and BAT in MFT by microscopic observation of histological changes (Fig. 1). The development of FALC was quantified by calculating the ratio of FALC area to total MFT area (Fig. 2). The ratio of FALC area to total MFT area in M. pulmonis-infected B6 mice was significantly lower $(P<0.005)$ than broth-inoculated control B6 mice but that in $M$. pulmonis-infected D2 mice was significantly higher $(P<0.005)$ than broth-inoculated control D2 mice (Fig. 2A). Interestingly, the ratios of FALC area to total MFT area in both SeV-infected B6 and D2 mice were significantly higher $(P<0.005)$ than broth-inoculated control B6 and D2 mice (Fig. 2A).

It was found that the ratio of BAT area to total MFT area was significantly increased $(P<0.005)$ in $M$. pulmonis-infected D2 mice when compared with broth-inoculated control D2 mice, but not in $M$. pulmonis-infected B6 mice when compared with brothinoculated control B6 mice (Fig. 2B). However, the ratios of BAT area to total MFT area were significantly increased $(P<0.005)$ in both SeV-infected B6 and D2 mice when compared with broth-inoculated control B6 and D2 mice, respectively (Fig. 2B).

These results indicated that M. pulmonis infection caused development of FALC and BAT in the MFT in D2 mice only, whereas $\mathrm{SeV}$ infection did it in both B6 and D2 mice.

\section{Development of BAT in 3 different adipose tissues in infected B6 and D2 mice}

To examine the increase in the amount of BAT in iBAT and the increase in browning of ingWAT and perigoWAT, these tissues were collected from M. pulmonis- and SeV-infected B6 and D2 mice. In broth-inoculated control B6 and D2 mice, no specific difference was observed in the histological appearances of iBAT, ingWAT, and perigoWAT. The iBAT displayed brown adipocytes containing multilocular lipid droplets (Fig. 3Aa and 3Ab). Both ingWAT and perigoWAT displayed white adipocytes containing large unilocular lipid droplets (Fig. 3Ba, 3Bb, 3Ca and 3Cb). The brown adipocytes of M. pulmonis- and SeV-infected D2 mice were increased in iBAT (Fig. 3Ad and 3Af). SeV-infected B6 mice showed an increase in brown adipocytes in iBAT but not in M. pulmonis-infected B6 mice (Fig. 3Ac and 3Ce). In ingWAT, beige adipocytes containing multilocular lipid droplets were found after M. pulmonis infection in D2 mice (Fig. 3Bd) but not in SeV-infected B6 and D2 mice (Fig. 3Be and 3Bf). In contrast, beige adipocytes were not seen in perigoWAT after M. pulmonis and SeV infection (Fig. 3Cc-f). Following these histological observations, the ratio of BAT area to total adipose tissue area was calculated. The ratio of BAT area to total iBAT area was significantly higher in the M. pulmonis-infected D2 mice $(P<0.01)$, SeV-infected D2 mice $(P<0.01)$, and SeV-infected B6 mice $(P<0.05)$ but lower in M. pulmonis-infected B6 mice when compared with broth-inoculated control mice (Fig. 4A). In both ingWAT and perigoWAT, the control group showed a very low ratio of BAT area to total WAT area. (Fig. 4B and 4C). Of note, only in M. pulmonis-infected D2 mice showed a significantly higher ratio of BAT area to total ingWAT area when compared to the control and the other infected groups $(P<0.01)$.

\section{Quantification of Ucpl gene expression in adipose tissues in infected B6 and D2 mice}

To quantify the numbers of brown and beige adipocytes in the iBAT, ingWAT, and perigoWAT, we examined the expression of the Ucp 1 gene. Between broth-inoculated control B6 and D2 mice showed no statistical difference in Ucp1 gene expression in the iBAT, whereas the infection significantly enhanced Ucpl gene expression in the iBAT of both M. pulmonis- and SeV-infected D2 mice $(P<0.01$ and $P<0.05$, respectively) but not in infected B6 mice (Fig. 5A). In addition, $M$. pulmonis-infected D2 mice only showed a statistically significant increase in Ucpl gene expression in ingWAT as compared with broth-inoculated control mice $(P<0.01)$ (Fig. 5B). All groups of infected mice showed no statistical difference in Ucpl gene expression in the perigoWAT as compared with broth-inoculated control groups (Fig. 5C). 

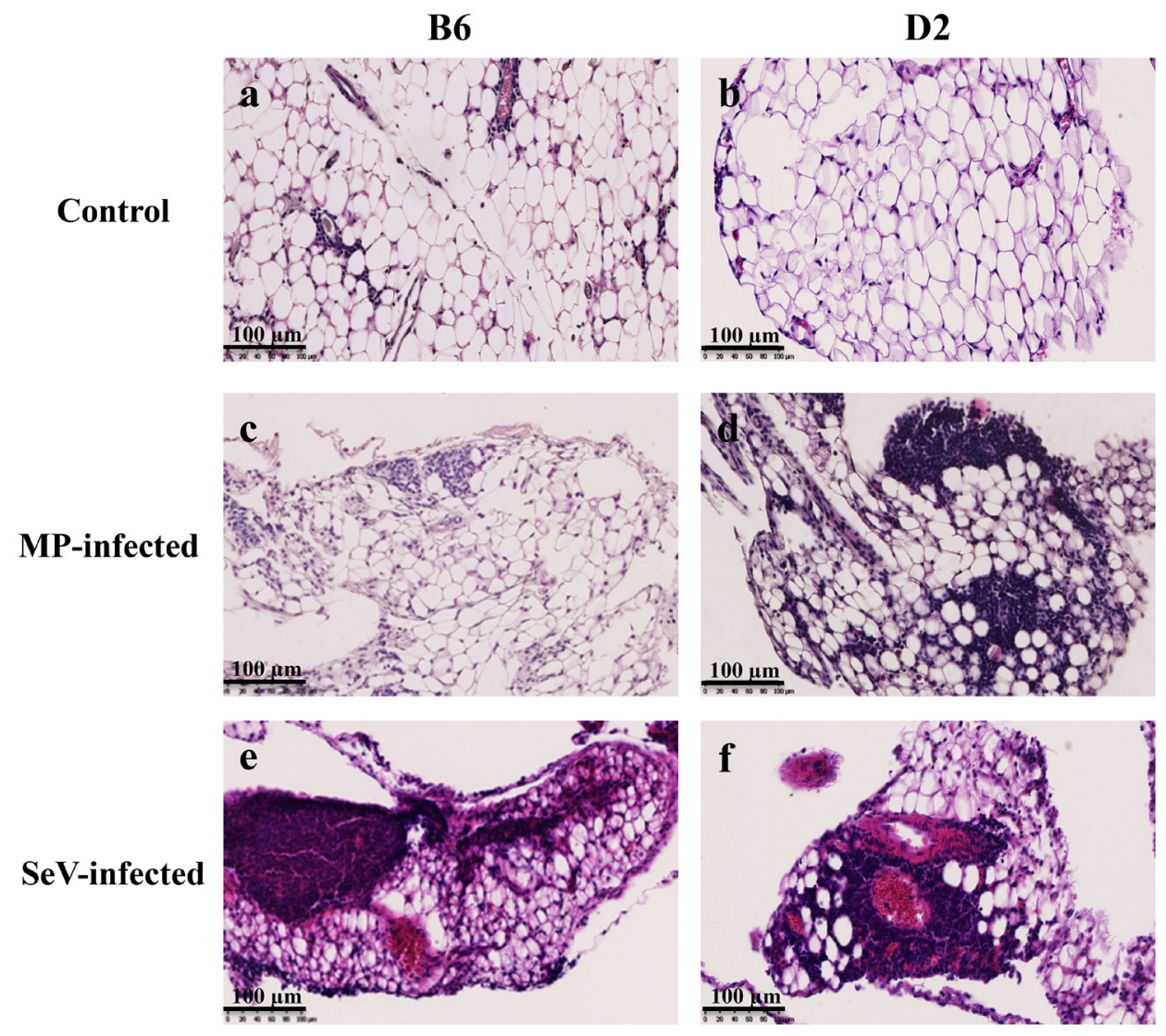

Fig. 1. The representative of histological features of fat-associated lymphoid clusters (FALCs) in mediastinal fat tissues (MFTs) in infected C57BL/6 (B6), infected DBA/2 (D2), and control mice after Mycoplasma (M.) pulmonis and Sendai virus (SeV) infection. Control (a and b), M. pulmonis infected (c and d), and SeV-infected (e and $\mathrm{f}$ ) mice ( $\mathrm{n}=4-6$ per group). Mononuclear cells accumulate in the MFT. Larger areas of FALCs are visible in M. pulmonis infected D2 mice (d) compared with M. pulmonis infected B6 (c) and control mice (a and b). However, larger areas of FALCs are visible in both SeV-infected mice (e and f) compared with control mice (a and b). All scale bars indicate $100 \mu \mathrm{m}$.

(A)

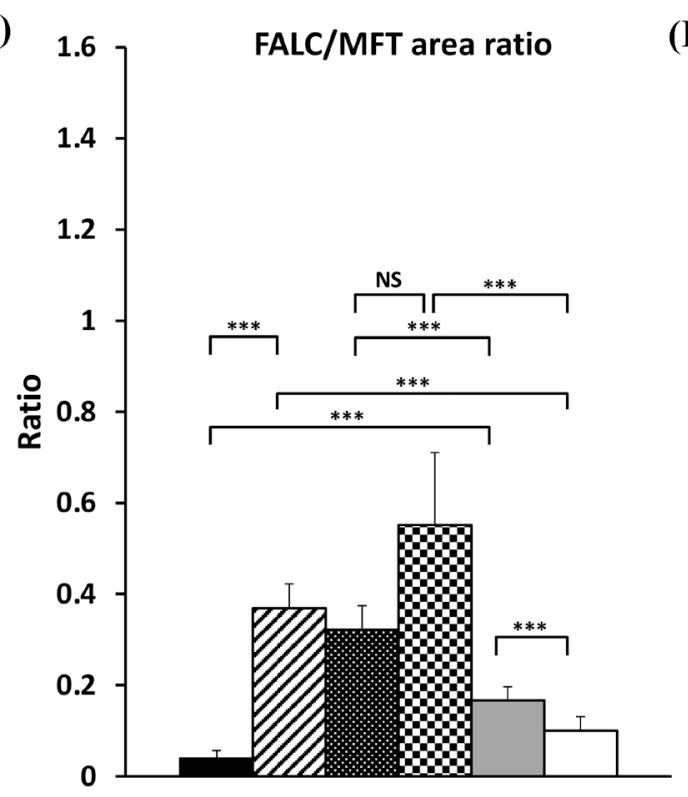

(B)

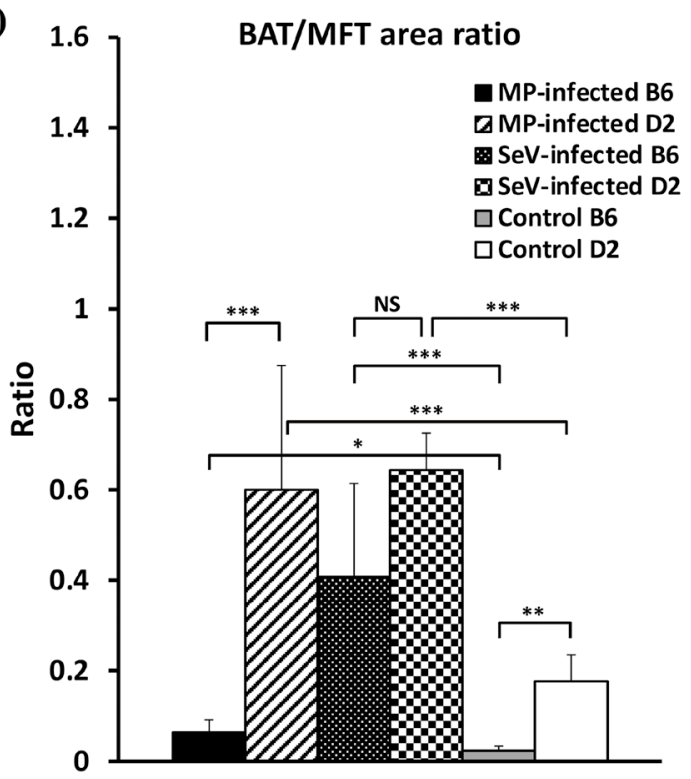

Fig. 2. Ratio of fat-associated lymphoid clusters (FALCs) area in the total mediastinal fat tissues (MFTs) area (A) and brown adipose tissue (BAT) area in the total MFT area (B) in the haematoxylin and eosin (HE)-stained sections in infected C57BL/6 (B6), infected DBA/2 (D2), and control mice after Mycoplasma (M.) pulmonis and Sendai virus ( $\mathrm{SeV}$ ) infection ( $\mathrm{n}=4-6$ per group). Values are mean $\pm \mathrm{SE}$ of the ratio. $*$, ** and *** indicate $P<0.05, P<0.01$, and $P<0.005$, respectively. NS; not significantly different. 
A iBAT

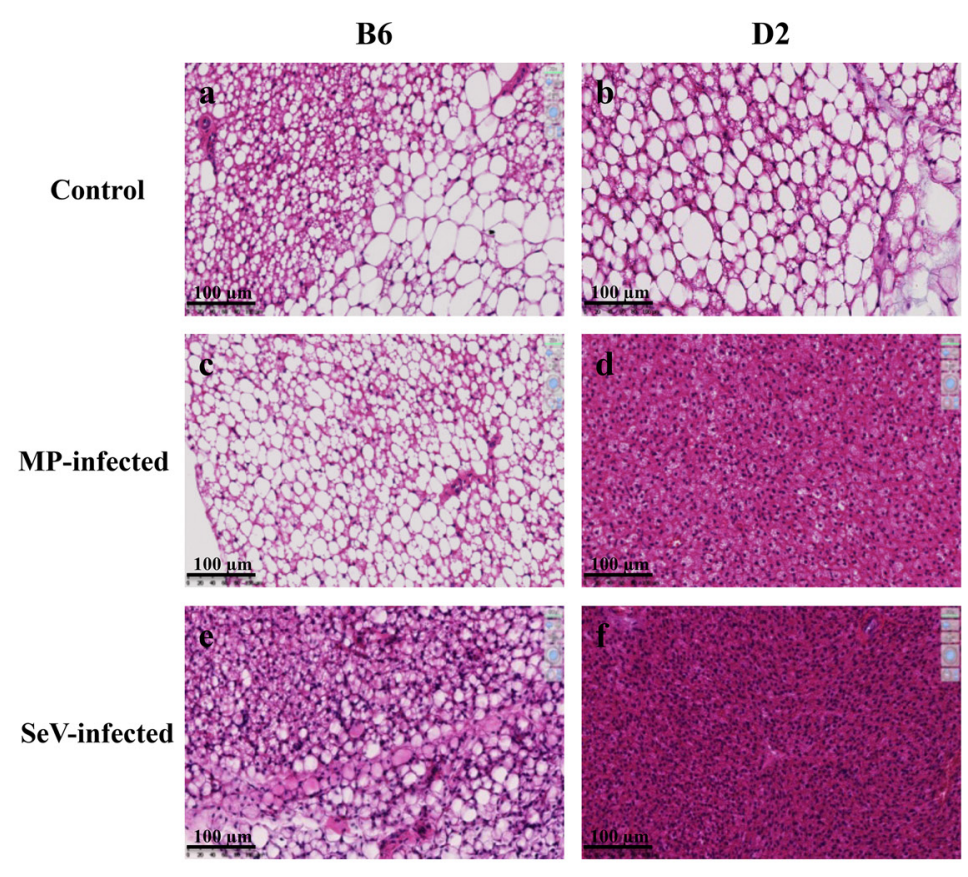

C perigoWaT

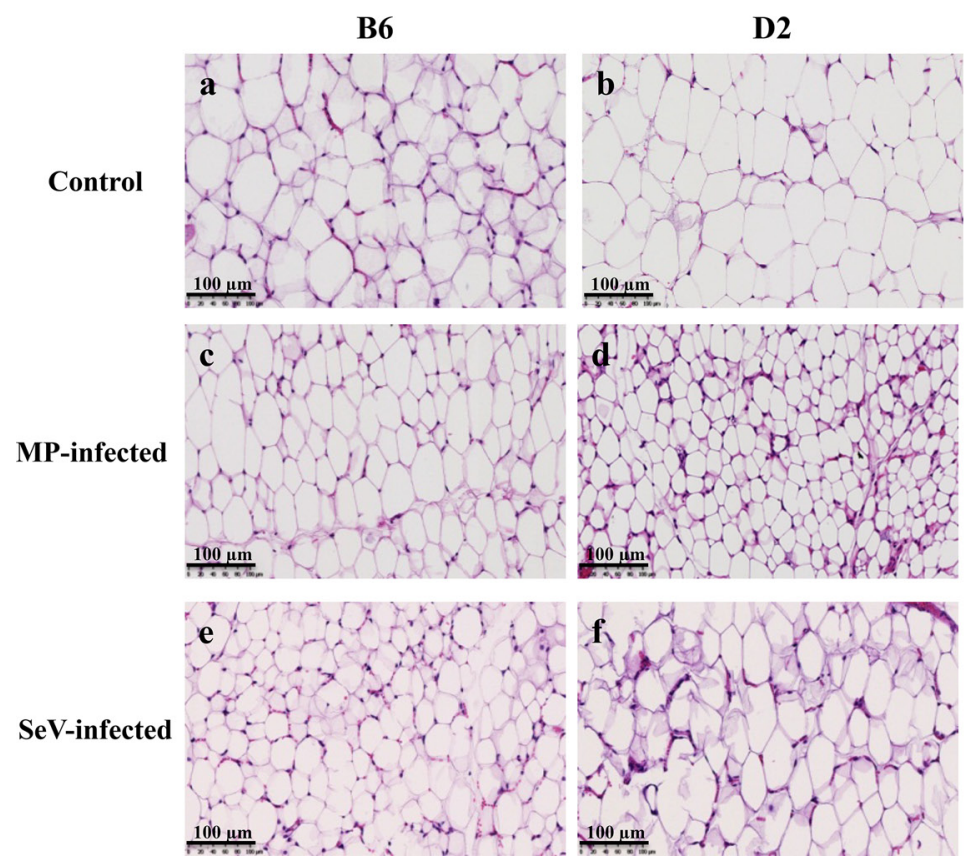

\section{B ingWAT}
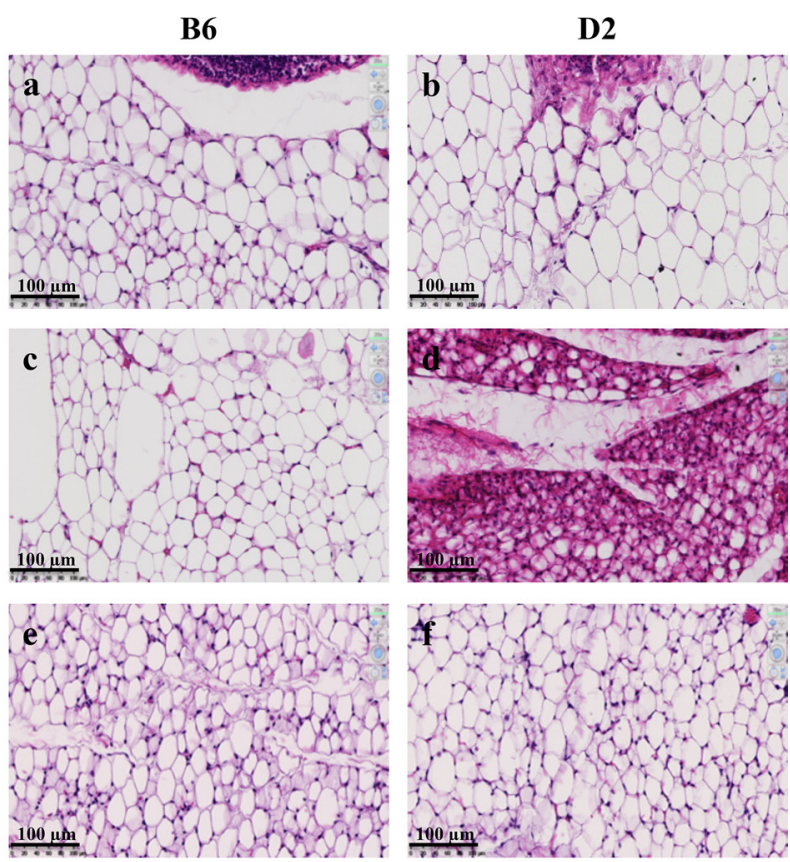

Fig. 3. The representative of histopathological observation of adipose tissue sections in infected C57BL/6 (B6), infected DBA/2 (D2), and control mice after Mycoplasma (M.) pulmonis and Sendai virus (SeV) infection. Control (a-b), M. pulmonis-infected (c-d), and SeV-infected (e-f) mice ( $\mathrm{n}=4-6$ per group). For $M$. pulmonis infection, $M$. pulmonis-infected D2 mice showed increasing of beige and/or brown adipose tissue in interscapular brown adipose tissue (iBAT) (3Ad) and inguinal WAT (ingWAT) (3Bd) compared to control mice (3Ab and $3 \mathrm{Bb})$. Conversely, M. pulmonis-infected B6 mice did not show increasing of beige and/or brown adipose tissue in iBAT (3Ac), ingWAT (3Bc), and perigonadal WAT (perigoWAT) (3Cc) compared to control mice (3Aa, 3Ba, and 3Ca). For $\mathrm{SeV}$ infection, SeV-infected D2 mice showed increasing of beige and/or brown adipose tissue in only iBAT (3Af) compared to control mice (3Ab). Interestingly, SeVinfected B6 mice showed a mild increase of beige and/or brown adipose tissue in iBAT (3Ae) compared to control mice (3Aa). All scale bars indicate $100 \mu \mathrm{m}$.

\section{Body temperature in infected B6 and D2 mice}

Because BAT is well known thermogenic tissues, it was considered that the increase in BAT contributed to the fever by pathogens infection. However, as shown in Table 1, body temperature was not changed after infection of M. pulmonis and SeV in both B6 and D2 mice.

\section{DISCUSSION}

Adipose tissues in mammals are categorized into WAT and BAT in which cellular morphology, cell functions, and tissue distribution are different. WAT plays a major role in energy reservation, while BAT mainly relates to the thermoregulation of the body. One interesting function of adipose tissue is the response to the infection, especially the pathogens that cause pneumonia. We have previously reported that D2 mice are susceptible to pathogens causing pneumonia, M. pulmonis and SeV, whereas B6 mice 

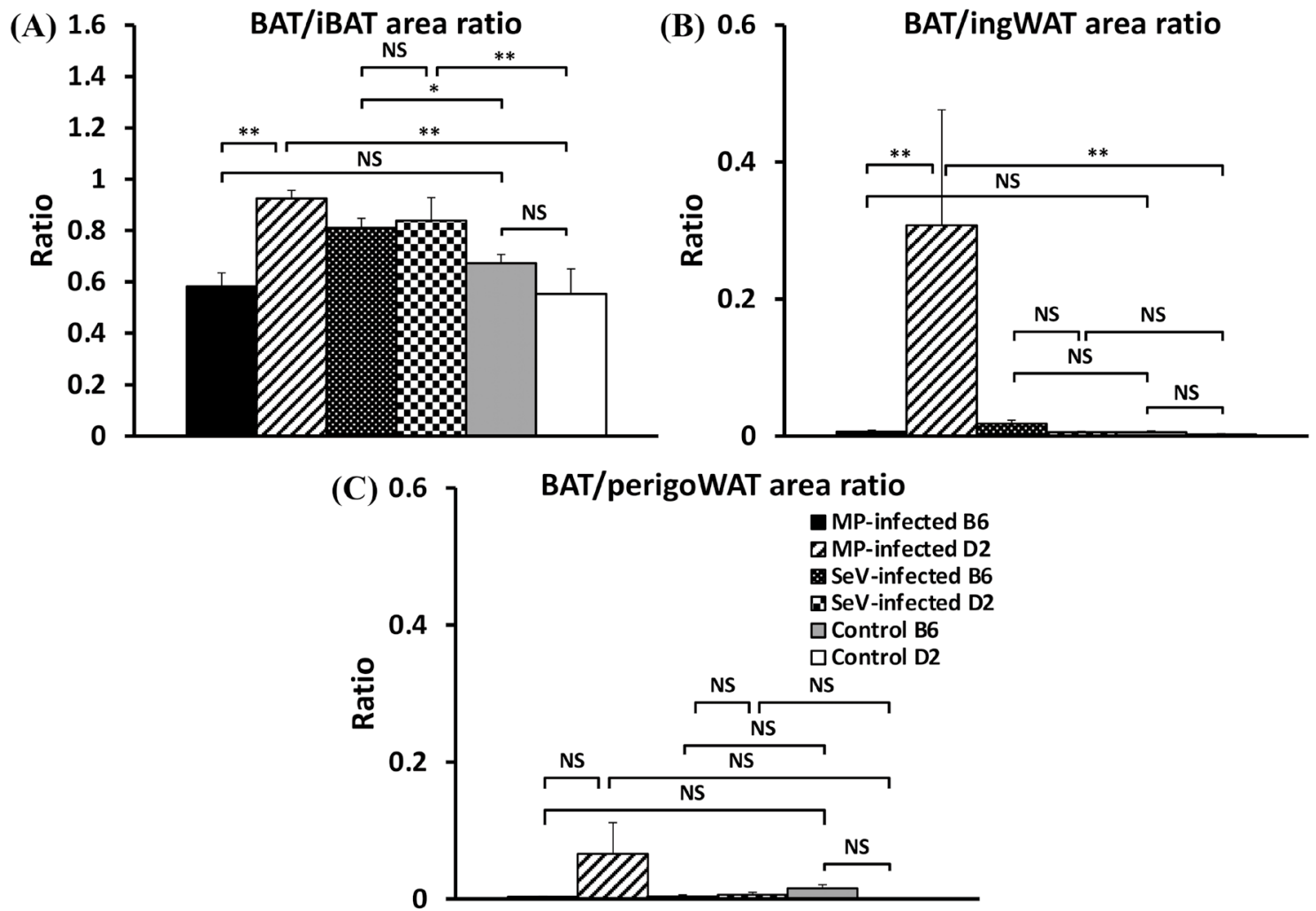

Fig. 4. Ratio of brown adipose tissue (BAT) area in the total interscapular BAT (iBAT) area (A), BAT area in the total inguinal WAT (ingWAT) area (B), and BAT area in the total perigonadal WAT (perigoWAT) area (C) in the haematoxylin and eosin (HE)-stained sections in infected C57BL/6 (B6), infected DBA/2 (D2), and control mice after Mycoplasma (M.) pulmonis and Sendai virus (SeV) infection ( $\mathrm{n}=4-6$ per group). Values are mean \pm SE of the ratio. * and ** indicate $P<0.05$ and $P<0.01$, respectively. NS; not significantly different.

are resistant to them. Furthermore, morphological alteration of MFT was seen after infection of M. pulmonis in D2 mice but not in B6 mice. In this study, we aimed to exhibit the difference in adipose tissue response in other areas, including iBAT, ingWAT, and perigoWAT between resistant strain, B6 and susceptible strain, D2 after challenging them with M. pulmonis and SeV. Compared with B6 mice, D2 mice showed an increase in FALC in MFT, an increase in BAT in both iBAT and ingWAT after M. pulmonis and $\mathrm{SeV}$ infection. The results of this study indicate that pneumonia caused by $M$. pulmonis and $\mathrm{SeV}$ infection induces browning of adipocyte, suggesting that BAT plays a role in pathogen infection and inflammation.

In our previous study, M. pulmonis-infected mice showed infiltration of many inflammatory cells such as neutrophils, macrophages, and lymphocytes into the lung and the number of FALC in MFT also increased, which consisted of mainly T and B cells [5]. The response in MFT implied that adipose tissue may play an important role in the infection. Therefore, in this study, the response of adipose tissues was investigated and it was found that a significant reduction in lipid droplets, an increase in the beige/ brown adipocytes development from white adipocytes, and an increase in the levels of immune cells in adipose tissue during $M$. pulmonis and $\mathrm{SeV}$ infection in adipose tissues including MFT (Figs. 1, 2B, 3A and 3B). However, clear FALCs were not observed in other adipose tissues unlike MFT (Fig. 3A-C). Therefore, an increase in FALC after infection seems to be specific to MFT. Since an increase in the beige/brown adipocytes development after infection was evident in this study, we reexamined relation between FALC/MFT and BAT/MFT using histology sections made in our previous paper [5]. Although both ratios were higher in infected D2 mice than infected B6 mice, the time course (7, 14, and 21 d.p.i) of both ratios were not necessarily consistent. The increasing of immune cells in lung and MFT and development of beige/brown adipocytes in adipose tissues collated with many studies which clarified that cytokines released from immune cells could activate white adipocyte browning [13, 21, 29].

In general, adipose tissue has been considered to store the surplus energy; however, in the past decades, increasing of evidence strongly suggests that adipose tissue has functions related to endocrine and immune systems. Adipose tissue is composed of many cell types, which contains pre-adipocytes and adipocytes, stromal vascular cells, immune cells, and endothelial cells. Immature haematopoietic cells are found in adipose tissue, suggesting that adipose tissue might be a place for the immune cell formation and maturation [20]. In addition, immune cells in adipose tissue are derived from bone marrow and migrate into the adipose tissue [30], where they may perform many functions, such as angiogenesis, apoptotic cell clearance, and remodeling of extracellular matrix [24]. Moreover, these immune cells in adipose tissue may participate in combating or raising infection. Both lipolysis and lipogenesis differentially regulate the secretion of adipokines, such as adiponectin, leptin, TNF- $\alpha$, IL-6, and IL-10, may result in imbalance of the host immunity and cause resistance or susceptibility to the infection. The macrophages migrated into adipose 

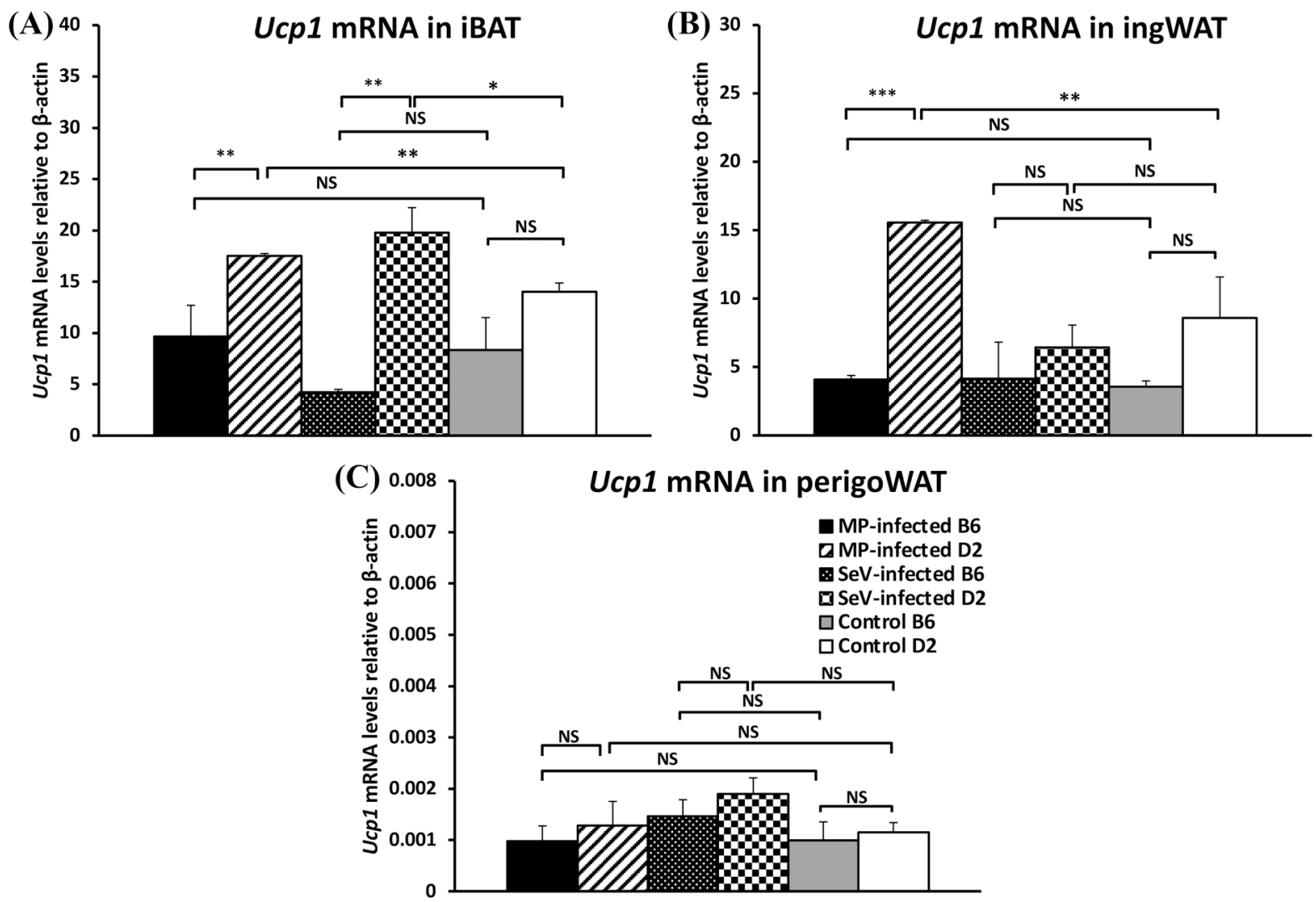

Fig. 5. Ucp1 mRNA levels in 3 different adipose tissues. Ucp1 mRNA levels (expressed per Actb) in interscapular brown adipose tissue (iBAT) (A), inguinal WAT (ingWAT) (B), and perigonadal WAT (perigoWAT) (C) in infected C57BL/6 (B6), infected DBA/2 (D2), and control mice after Mycoplasma (M.) pulmonis and Sendai virus (SeV) infection ( $\mathrm{n}=4-6$ per group). Values are mean $\pm \mathrm{SE}$ of the ratio. $*$, ** and $* * *$ indicate $P<0.05, P<0.01$, and $P<0.005$, respectively. NS; not significantly different.

Table 1. Body temperature after Mycoplasma (M.) pulmonis and Sendai virus infection in C57BL/6 (B6) and DBA/2 (D2) mice

\begin{tabular}{|c|c|c|c|c|}
\hline \multirow{3}{*}{$\begin{array}{l}\text { Days post } \\
\text { infection }\end{array}$} & \multicolumn{4}{|c|}{ Body temperature $\left({ }^{\circ} \mathrm{C}\right)$} \\
\hline & \multicolumn{2}{|c|}{ M. pulmonis infection } & \multicolumn{2}{|c|}{ Sendai virus infection } \\
\hline & B6 & D2 & B6 & D2 \\
\hline 0 & $36.45 \pm 0.12$ & $36.42 \pm 0.12$ & $36.47 \pm 0.09$ & $36.40 \pm 0.14$ \\
\hline 1 & $36.45 \pm 0.12$ & $36.57 \pm 0.05$ & $36.50 \pm 0.08$ & $36.47 \pm 0.15$ \\
\hline 2 & $36.50 \pm 0.14$ & $36.37 \pm 0.09$ & $36.52 \pm 0.09$ & $36.45 \pm 0.17$ \\
\hline 3 & $36.42 \pm 0.12$ & $36.37 \pm 0.09$ & $36.50 \pm 0.18$ & $36.45 \pm 0.19$ \\
\hline 4 & $36.45 \pm 0.23$ & $36.35 \pm 0.10$ & $36.45 \pm 0.10$ & $36.45 \pm 0.10$ \\
\hline 5 & $36.42 \pm 0.18$ & $36.40 \pm 0.14$ & $36.47 \pm 0.09$ & $36.47 \pm 0.05$ \\
\hline 6 & $36.42 \pm 0.15$ & $36.32 \pm 0.15$ & $36.40 \pm 0.08$ & $36.40 \pm 0.14$ \\
\hline 7 & $36.37 \pm 0.09$ & $36.35 \pm 0.10$ & $36.40 \pm 0.11$ & $36.32 \pm 0.05$ \\
\hline 8 & $36.27 \pm 0.25$ & $36.12 \pm 0.12$ & $36.35 \pm 0.05$ & $36.32 \pm 0.05$ \\
\hline 9 & $36.10 \pm 0.14$ & $36.15 \pm 0.19$ & ND & ND \\
\hline 10 & $35.97 \pm 0.22$ & $36.00 \pm 0.27$ & ND & ND \\
\hline 11 & $35.92 \pm 0.05$ & $36.02 \pm 0.18$ & ND & ND \\
\hline 12 & $36.02 \pm 0.20$ & $35.95 \pm 0.05$ & ND & ND \\
\hline 13 & $36.02 \pm 0.09$ & $35.97 \pm 0.05$ & ND & ND \\
\hline 14 & $35.97 \pm 0.15$ & $35.95 \pm 0.10$ & ND & ND \\
\hline
\end{tabular}

Data are shown as mean $\pm \mathrm{SD} ; \mathrm{n}=4-6$. ND: not determined. 
tissue can produce cytokines especially IL-1 $\beta$, which induce inflammation response. It has been reported that the injection of IL-1 $\beta$ suppressed cold-induced browning [9], indicating that these macrophages secrete inflammatory cytokines and suppress browning of WAT. Thus, we assumed that the low browning ability of iBAT and ingWAT occurred in M. pulmonis- and SeV-infected B6 mice may be due to the high level of IL-1 $\beta$ releasing from the macrophages. This hypothesis correlated with our recent finding, which showed increased IL-1 $\beta$ in BALF after the infection with M. pulmonis [5] and SeV [27]. In addition, many studies showed that even pre-adipocytes and adipocytes are responded to the invading microbes. For example, pre-adipocytes and adipocytes secrete numerous inflammatory cytokines such as TNF- $\alpha$ and are sensitive to lipopolysaccharide activation [14]. In our previous study, the level of TNF- $\alpha$ in BALF increased with decreasing body weight, which suggests that TNF- $\alpha$ may accelerate WAT lipolysis in the early stage of M. pulmonis and SeV infection. Moreover, release of many cytokines especially IL-6 has been shown the correlation with weight loss and survival rate [8], suggesting that IL-6 might induce weight loss in SeV and M. pulmonis infection by accelerating WAT lipolysis. On the other hand, the recent paper has indicated the possibility that the infected pathogen, influenza virus, located in adipose tissues and directly induced the change of WAT to BAT [2]. Because we did not examine the presence of pathogens in adipose tissues in this study, we cannot verify this possibility. The confirmation of these hypotheses needs further studies.

Microbial invasion activates the immune cells by increasing their population to eliminate pathogens and simultaneously may stimulate the expansion of BAT in response to the infection. On the other hand, the reduction of immune response in SeV-and $M$. pulmonis-infected B6 mice may be linked to a decrease in immune function by excessively expanded adipose tissue. The expansion of adipose tissue decreases pre-adipocytes and other adipogenic precursors [11] that have antimicrobial properties. This may collectively contribute to the reduction of unnecessary immune response in SeV-and M. pulmonis-infected B6 mice or depletion of adipocyte-related immune cells in SeV- and M. pulmonis-infected D2 mice. We have shown that lipolysis and browning of WAT increased pathogens burden, which was associated with foamy macrophages and anti-inflammatory signaling. Thus, an altered balance between pro- and anti-inflammatory signaling in adipose tissue and the lung is an important feature of the link between adipose tissue pathophysiology and pulmonary pathology in the context of wasting during SeV and M. pulmonis infection.

Recent data suggest that there are two distinct types of brown fat, classical BAT derived from myogenic factor 5 lineage cells and UCP1-positive cells referred to as beige adipocytes that emerge in white fat from a non-myogenic factor 5 lineage [19, 31]. In our study, only one marker for both beige and brown fat-associated gene, the Ucpl gene, was used in this study and upregulation of the Ucpl gene were found in iBAT and ingWAT of SeV- and M. pulmonis-infected mice, but it was not necessarily consistent with the increase in BAT in the histopathology sections. Further study should be performed by using more markers associated with beige and brown fat to observe up- and/or down-regulation of the related genes in adipose tissues.

Pathogens infection usually causes a fever generated by increasing heat production and reducing heat loss. In the classical view, BAT is a key effector tissue that maintains metabolic homeostasis by adjusting energy expenditure and adaptive heat production. However, the contribution of increasing BAT to fever thermogenesis in systemic inflammation is unclear [16, 28]. In this study the increasing of BAT after M. pulmonis and SeV infection was found without the increasing of body temperature in the infected mice (Table 1), suggesting that BAT induction after M. pulmonis and SeV infection may not relate to thermogenesis for fever. Generally, the conversion of white adipocyte to beige/brown adipocyte was induced by cold exposure [17]. Interestingly, our results suggest that an increase in beige/brown adipocytes after M. pulmonis and $\mathrm{SeV}$ infection did not play an important role in fever induction in response to the infection. However, confirmation of this result needs a further study to clarify the function of changing WAT to BAT after pathogens infection and underlying mechanisms for it.

In conclusion, our results offer new pieces of information regarding the subtle interplay between pathogens infection that cause pneumonia such as M. pulmonis and $\mathrm{SeV}$ and their hosts. The findings in combination with the increasing of WAT lipolysis and browning-associated gene in inguinal WAT of M. pulmonis and SeV infection might be caused by cytokines releasing from adipocytes. These considerations underscore the need to recognize and further investigate the interaction of adipose tissue and pathogens infections.

POTENTIAL CONFLICTS OF INTEREST. The authors have nothing to disclose.

ACKNOWLEDGMENTS. We thank Dr. Nobuhito Hayashimoto, Central Institute for Experimental Animals, Kawasaki, Japan, for the kind gift of M. pulmonis stock, CIEA-NH strain and Ms. Jussiaea Bariuan, Laboratory of Biochemistry, Graduate School of Veterinary Medicine, Hokkaido University, for the assistance in mRNA analysis. Tussapon Boonyarattanasoonthorn was supported by a scholarship from the Ministry of Education, Culture, Sports, Science and Technology, Japan. Keisuke Sato was supported by the World-leading Innovative and Smart Education (WISE) Program (1801) from the Ministry of Education, Culture, Sports, Science, and Technology, Japan.

\section{REFERENCES}

1. Anderson, E. K., Gutierrez, D. A. and Hasty, A. H. 2010. Adipose tissue recruitment of leukocytes. Curr. Opin. Lipidol. 21: 172-177. [Medline] [CrossRef]

2. Ayari, A., Rosa-Calatrava, M., Lancel, S., Barthelemy, J., Pizzorno, A., Mayeuf-Louchart, A., Baron, M., Hot, D., Deruyter, L., Soulard, D., Julien, T., Faveeuw, C., Molendi-Coste, O., Dombrowicz, D., Sedano, L., Sencio, V., Le Goffic, R., Trottein, F. and Wolowczuk, I. 2020. Influenza infection rewires energy metabolism and induces browning features in adipose cells and tissues. Commun. Biol. 3: 237. [Medline] [CrossRef] 
3. Ayyappan, J. P., Vinnard, C., Subbian, S. and Nagajyothi, J. F. 2018. Effect of Mycobacterium tuberculosis infection on adipocyte physiology. Microbes Infect. 20: 81-88. [Medline] [CrossRef]

4. Berg, A. H. and Scherer, P. E. 2005. Adipose tissue, inflammation, and cardiovascular disease. Circ. Res. 96: 939-949. [Medline] [CrossRef]

5. Boonyarattanasoonthorn, T., Elewa, Y. H. A., Tag-El-Din-Hassan, H. T., Morimatsu, M. and Agui, T. 2019. Profiling of cellular immune responses to Mycoplasma pulmonis infection in C57BL/6 and DBA/2 mice. Infect. Genet. Evol. 73: 55-65. [Medline] [CrossRef]

6. Bouzid, F., Brégeon, F., Poncin, I., Weber, P., Drancourt, M. and Canaan, S. 2017. Mycobacterium canettii infection of adipose tissues. Front. Cell. Infect. Microbiol. 7: 189. [Medline] [CrossRef]

7. Fantuzzi, G. 2005. Adipose tissue, adipokines, and inflammation. J. Allergy Clin. Immunol. 115: 911-919, quiz 920. [Medline] [CrossRef]

8. Fearon, K. C., Glass, D. J. and Guttridge, D. C. 2012. Cancer cachexia: mediators, signaling, and metabolic pathways. Cell Metab. 16: $153-166$. [Medline] [CrossRef]

9. Goto, T., Naknukool, S., Yoshitake, R., Hanafusa, Y., Tokiwa, S., Li, Y., Sakamoto, T., Nitta, T., Kim, M., Takahashi, N., Yu, R., Daiyasu, H., Seno, S., Matsuda, H. and Kawada, T. 2016. Proinflammatory cytokine interleukin-1 $\beta$ suppresses cold-induced thermogenesis in adipocytes. Cytokine 77: 107-114. [Medline] [CrossRef]

10. Halberg, N., Wernstedt-Asterholm, I. and Scherer, P. E. 2008. The adipocyte as an endocrine cell. Endocrinol. Metab. Clin. North Am. 37: 753-768, $\mathrm{x}-\mathrm{xi}$. [Medline] [CrossRef]

11. Isakson, P., Hammarstedt, A., Gustafson, B. and Smith, U. 2009. Impaired preadipocyte differentiation in human abdominal obesity: role of Wnt, tumor necrosis factor- $\alpha$, and inflammation. Diabetes 58: 1550-1557. [Medline] [CrossRef]

12. Kawai, S., Takagi, Y., Kaneko, S. and Kurosawa, T. 2011. Effect of three types of mixed anesthetic agents alternate to ketamine in mice. Exp. Anim. 60: 481-487. [Medline] [CrossRef]

13. Kristóf, E., Klusóczki, Á., Veress, R., Shaw, A., Combi, Z. S., Varga, K., Győry, F., Balajthy, Z., Bai, P., Bacso, Z. and Fésüs, L. 2019. Interleukin-6 released from differentiating human beige adipocytes improves browning. Exp. Cell Res. 377: 47-55. [Medline] [CrossRef]

14. Lin, Y., Lee, H., Berg, A. H., Lisanti, M. P., Shapiro, L. and Scherer, P. E. 2000. The lipopolysaccharide-activated toll-like receptor (TLR)-4 induces synthesis of the closely related receptor TLR-2 in adipocytes. J. Biol. Chem. 275: 24255-24263. [Medline] [CrossRef]

15. Nagajyothi, F., Weiss, L. M., Silver, D. L., Desruisseaux, M. S., Scherer, P. E., Herz, J. and Tanowitz, H. B. 2011. Trypanosoma cruzi utilizes the host low density lipoprotein receptor in invasion. PLoS Negl. Trop. Dis. 5: e953. [Medline] [CrossRef]

16. Okamatsu-Ogura, Y., Kitao, N., Kimura, K. and Saito, M. 2007. Brown fat UCP1 is not involved in the febrile and thermogenic responses to IL-1 $\beta$ in mice. Am. J. Physiol. Endocrinol. Metab. 292: E1135-E1139. [Medline] [CrossRef]

17. Okamatsu-Ogura, Y., Fukano, K., Tsubota, A., Nio-Kobayashi, J., Nakamura, K., Morimatsu, M., Sakaue, H., Saito, M. and Kimura, K. 2017. Cellcycle arrest in mature adipocytes impairs BAT development but not WAT browning, and reduces adaptive thermogenesis in mice. Sci. Rep. 7: 6648. [Medline] [CrossRef]

18. Ouchi, N., Parker, J. L., Lugus, J. J. and Walsh, K. 2011. Adipokines in inflammation and metabolic disease. Nat. Rev. Immunol. 11: 85-97. [Medline] [CrossRef]

19. Peirce, V., Carobbio, S. and Vidal-Puig, A. 2014. The different shades of fat. Nature 510: 76-83. [Medline] [CrossRef]

20. Poglio, S., De Toni-Costes, F., Arnaud, E., Laharrague, P., Espinosa, E., Casteilla, L. and Cousin, B. 2010. Adipose tissue as a dedicated reservoir of functional mast cell progenitors. Stem Cells 28: 2065-2072. [Medline] [CrossRef]

21. Qiu, Y., Nguyen, K. D., Odegaard, J. I., Cui, X., Tian, X., Locksley, R. M., Palmiter, R. D. and Chawla, A. 2014. Eosinophils and type 2 cytokine signaling in macrophages orchestrate development of functional beige fat. Cell 157: 1292-1308. [Medline] [CrossRef]

22. Rajala, M. W. and Scherer, P. E. 2003. Minireview: The adipocyte - at the crossroads of energy homeostasis, inflammation, and atherosclerosis. Endocrinology 144: 3765-3773. [Medline] [CrossRef]

23. Scherer, P. E. 2006. Adipose tissue: from lipid storage compartment to endocrine organ. Diabetes 55: 1537-1545. [Medline] [CrossRef]

24. Schipper, H. S., Prakken, B., Kalkhoven, E. and Boes, M. 2012. Adipose tissue-resident immune cells: key players in immunometabolism. Trends Endocrinol. Metab. 23: 407-415. [Medline] [CrossRef]

25. Schmid, A., Karrasch, T., Thomalla, M., Schlegel, J., Salzberger, B., Schäffler, A. and Hanses, F. 2017. Innate immunity of adipose tissue in rodent models of local and systemic Staphylococcus aureus infection. Mediators Inflamm. 2017: 5315602. [Medline] [CrossRef]

26. Simon, A. Y., Moritoh, K., Torigoe, D., Asano, A., Sasaki, N. and Agui, T. 2009. Multigenic control of resistance to Sendai virus infection in mice. Infect. Genet. Evol. 9: 1253-1259. [Medline] [CrossRef]

27. Simon, A. Y., Sasaki, N., Ichii, O., Kajino, K., Kon, Y. and Agui, T. 2011. Distinctive and critical roles for cellular immunity and immuneinflammatory response in the immunopathology of Sendai virus infection in mice. Microbes Infect. 13: 783-797. [Medline] [CrossRef]

28. Szentirmai, É. and Kapás, L. 2018. Brown adipose tissue plays a central role in systemic inflammation-induced sleep responses. PLoS One 13: e0197409. [Medline] [CrossRef]

29. Villarroya, F., Cereijo, R., Villarroya, J., Gavaldà-Navarro, A. and Giralt, M. 2018. Toward an understanding of how immune cells control brown and beige adipobiology. Cell Metab. 27: 954-961. [Medline] [CrossRef]

30. Weisberg, S. P., McCann, D., Desai, M., Rosenbaum, M., Leibel, R. L. and Ferrante, A. W. Jr. 2003. Obesity is associated with macrophage accumulation in adipose tissue. J. Clin. Invest. 112: 1796-1808. [Medline] [CrossRef]

31. Wu, J., Cohen, P. and Spiegelman, B. M. 2013. Adaptive thermogenesis in adipocytes: is beige the new brown? Genes Dev. 27: 234-250. [Medline] [CrossRef] 